\title{
EFFECTS OF RESIDUAL WATER IN A QUADRUPOLE ION TRAP ON THE PROTONATION SITES OF 4-AMINOBENZOIC ACID SUPPORTING INFORMATION
}

\author{
Rashmi Kumar, Ravikiran Yerabolu, and Hilkka I. Kenttämaa* \\ Chemistry Department, Purdue University, 560 Oval Drive, West Lafayette, IN 47907
}




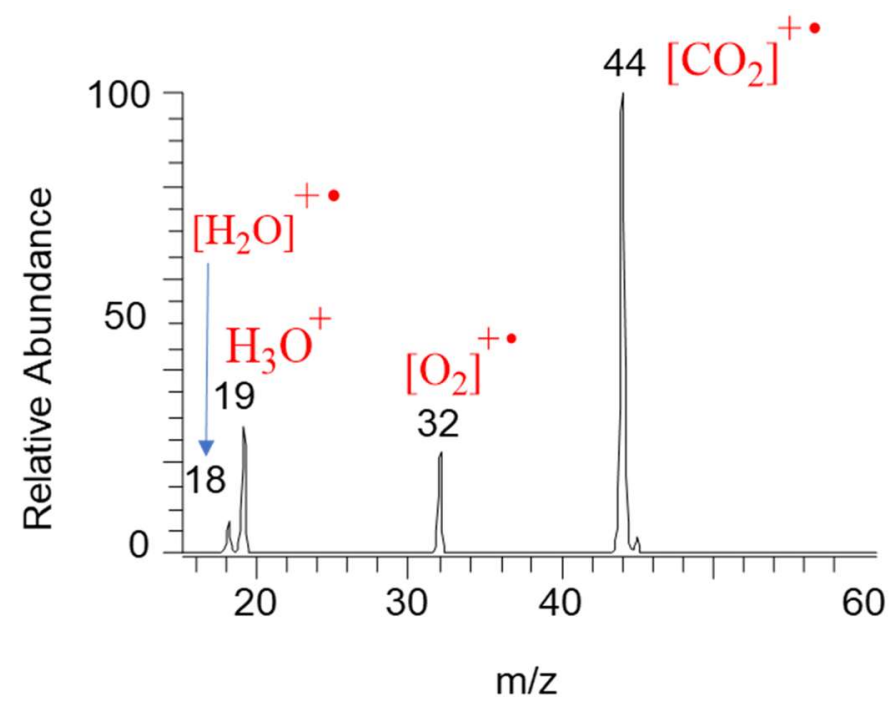

Figure S1: Water and oxygen detected in the linear quadrupole ion trap when the molecular ions of carbon dioxide (ionized using APCI) were isolated and allowed to react with residual molecules in the ion trap for $30 \mathrm{~ms}$. As $\mathrm{N}_{2}$ has a greater ionization energy $(15.6 \mathrm{eV})$ than $\mathrm{CO}_{2}(13.8 \mathrm{eV}), \mathrm{N}_{2}$ in the ion trap cannot be detected using this method.

\section{Experimental set-up for Figure S1}

$\mathrm{CO}_{2}$ gas instead of $\mathrm{N}_{2}$ was used as the sheath gas and auxiliary gas in atmospheric pressure chemical ionization $(\mathrm{APCl})$. Following parameters were used for $\mathrm{APCl}$ : vaporizer temperature: $300{ }^{\circ} \mathrm{C}$, capillary temperature: $250^{\circ} \mathrm{C}$, capillary voltage: $5 \mathrm{kV}$, sheath gas flow: 30 (arbitrary units), and auxiliary gas flow: 20 (arbitrary units). Molecular ions of $\mathrm{CO}_{2}$ were isolated and allowed to react with residual gases in the ion trap for $30 \mathrm{~ms}$.

\section{Results for Figure S1}

As shown in Figure $\mathrm{S} 1$, molecular ions of $\mathrm{H}_{2} \mathrm{O}$ and $\mathrm{O}_{2}$ as well as protonated water were observed in the linear quadrupole ion trap. This proves the presence of a considerable amount of water in the ion trap 


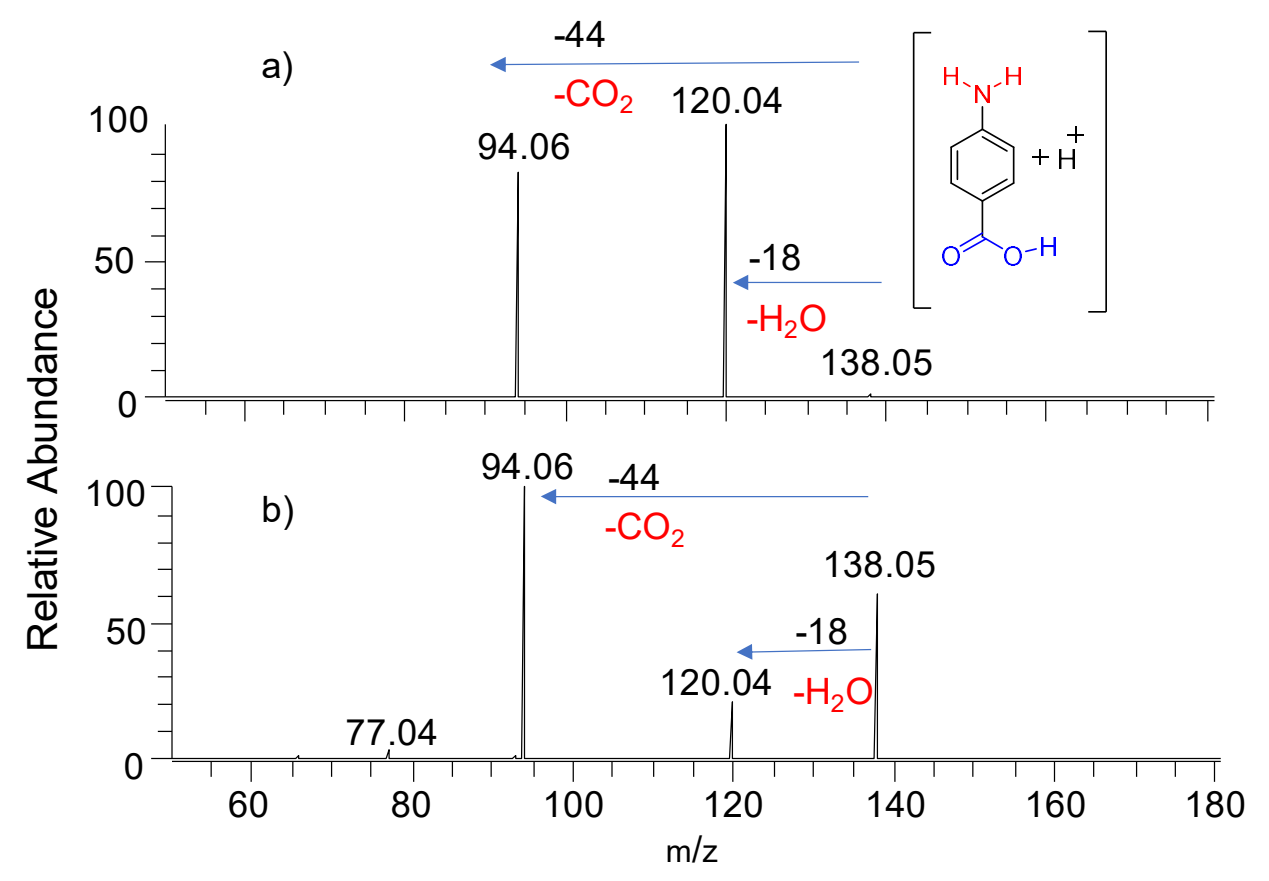

Figure S2. ITCAD products (collision energy: 35 arbitrary units) of protonated 4-aminobenzoic acid detected by the orbitrap when using a) 3-1 (v/v) methanol-water and b) 1-1 (v/v) acetonitrile-water solvent systems. 


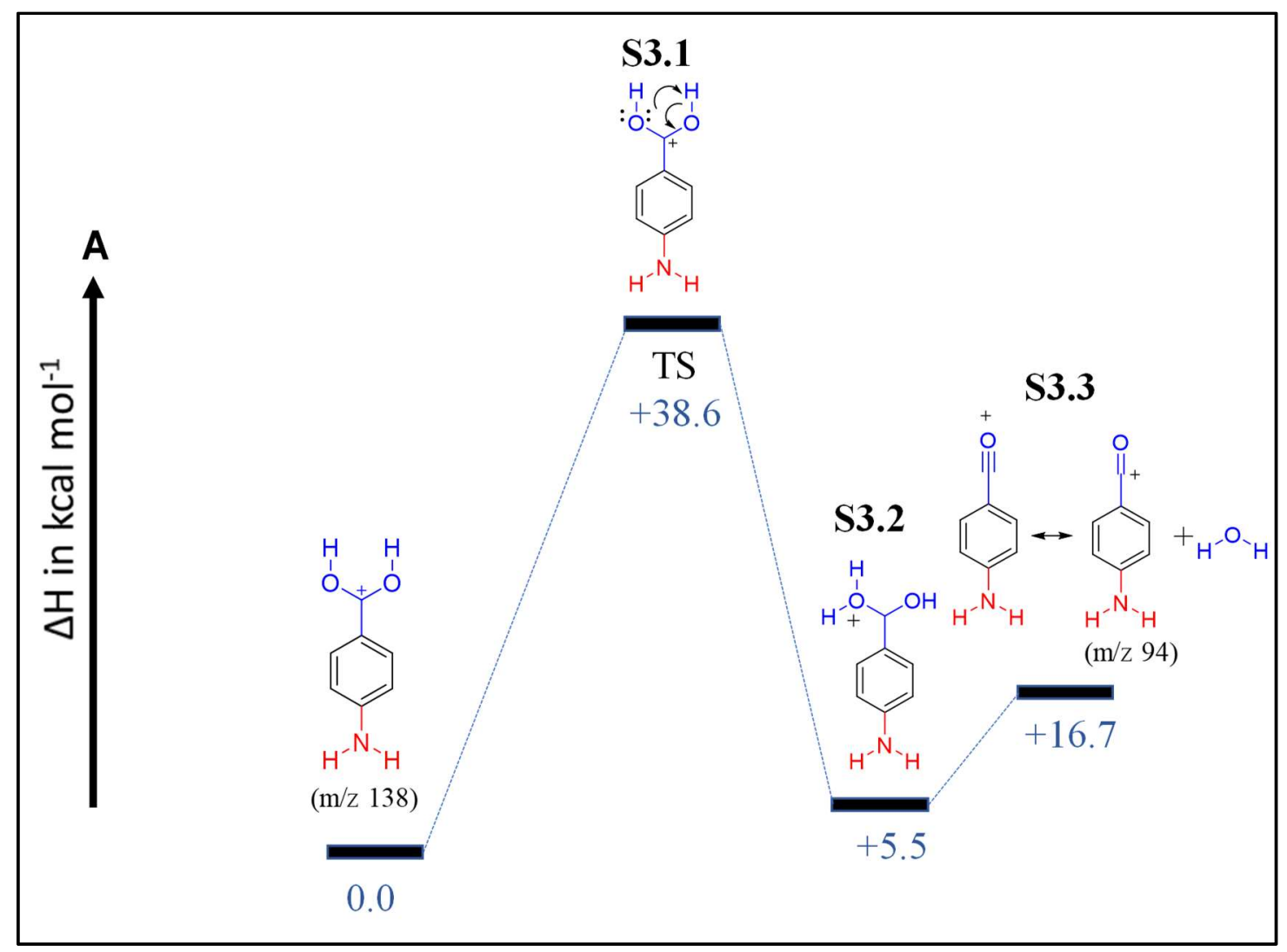

Figure S3. Calculated potential energy surface for $\mathrm{H}_{2} \mathrm{O}$ loss from the O-protomer of protonated 4-aminobenzoic acid.

\begin{tabular}{|l|l|l|l|}
\hline O-protomer of protonated & \multicolumn{4}{l|}{ M06-2X/6-311++G (d, p) } \\
\hline Enthalpy & \multicolumn{4}{l|}{} \\
\hline & \multicolumn{4}{l|}{ Cartesian Coordinates (Angstroms) } & z \\
\hline & $\mathrm{x}$ & $\mathrm{y}$ & \\
\hline C & 1.433189 & -1.22884 & -0.00954 \\
\hline C & 0.071403 & -1.22638 & -0.00985 \\
\hline C & -0.64601 & 0 & 0 \\
\hline C & 0.071403 & 1.226377 & 0.009849 \\
\hline
\end{tabular}




\begin{tabular}{|l|l|l|l|}
\hline C & 1.433189 & 1.228836 & 0.009539 \\
\hline C & 2.152291 & 0 & 0 \\
\hline H & 1.980938 & -2.16307 & -0.01787 \\
\hline H & -0.471 & -2.16349 & -0.01867 \\
\hline H & -0.471 & 2.163489 & 0.018668 \\
\hline C & 1.980938 & 2.16307 & 0.017874 \\
\hline O & -2.04318 & 0 & 0 \\
\hline O & -2.68646 & 1.137735 & -0.0305 \\
\hline N & -2.68646 & -1.13774 & 0.030502 \\
\hline H & 3.486266 & 0 & $-2 \mathrm{E}-06$ \\
\hline H & 4.010288 & -0.86112 & -0.00568 \\
\hline H & -3.64735 & 1.075922 & 0.047298 \\
\hline H & 4.010288 & 0.861117 & 0.005686 \\
\hline & -3.64735 & -1.07592 & -0.0473 \\
\hline
\end{tabular}

\begin{tabular}{|l|l|l|l|}
\hline S3.1 & \multicolumn{2}{l|}{ M06-2X/6-311++G $(\mathrm{d}, \mathrm{p})$} \\
\hline Enthalpy & \multicolumn{2}{|l|}{-476.245032 a.u } \\
\hline & \multicolumn{3}{|l|}{ Cartesian Coordinates (Angstroms) } \\
\hline & $\mathrm{x}$ & $\mathrm{y}$ & $\mathrm{z}$ \\
\hline C & 0.142006 & 1.267986 & -0.0373 \\
\hline
\end{tabular}




\begin{tabular}{|l|l|l|l|}
\hline C & 1.503566 & 1.224724 & -0.04437 \\
\hline C & 2.180661 & -0.02676 & -0.00379 \\
\hline C & 1.422551 & -1.23165 & 0.047118 \\
\hline C & 0.060471 & -1.18496 & 0.051613 \\
\hline C & -0.61165 & 0.063524 & 0.00422 \\
\hline H & -0.37922 & 2.217872 & -0.06691 \\
\hline H & 2.081401 & 2.140274 & -0.07938 \\
\hline H & 1.939418 & -2.18232 & 0.093854 \\
\hline H & -0.50161 & -2.10971 & 0.119429 \\
\hline N & 3.514946 & -0.0716 & -0.00876 \\
\hline C & -1.99965 & 0.166354 & 0.019263 \\
\hline O & -2.87403 & -1.00108 & -0.01501 \\
\hline O & -2.82809 & 1.073614 & 0.087529 \\
\hline H & -2.80672 & -1.59197 & -0.78656 \\
\hline H & 4.067731 & 0.771542 & -0.03656 \\
\hline H & 4.012306 & -0.94828 & 0.023322 \\
\hline H & & -0.05184 & \\
\hline
\end{tabular}

\begin{tabular}{|c|c|c|c|}
\hline S3.2 & \multicolumn{3}{|c|}{ M06-2X/6-311++G (d, p) } \\
\hline \multirow[t]{3}{*}{ Enthalpy } & \multicolumn{3}{|c|}{-476.297696 a.u } \\
\hline & \multicolumn{3}{|c|}{ Cartesian Coordinates (Angstroms) } \\
\hline & $x$ & $y$ & $z$ \\
\hline
\end{tabular}




\begin{tabular}{|l|l|l|l|}
\hline C & -0.1356 & -0.91029 & 0.000055 \\
\hline C & 1.187316 & -1.2333 & 0.000043 \\
\hline C & 2.186786 & -0.21925 & $-2 E-06$ \\
\hline C & 1.794984 & 1.150344 & $-3.2 \mathrm{E}-05$ \\
\hline C & 0.477324 & 1.489575 & -0.00002 \\
\hline C & -0.51366 & 0.461728 & 0.000023 \\
\hline H & -0.90666 & -1.66867 & 0.000091 \\
\hline H & 1.488723 & -2.27378 & 0.000068 \\
\hline H & 2.553906 & 1.923143 & $-6.3 \mathrm{E}-05$ \\
\hline H & 0.18158 & 2.531806 & $-4.3 \mathrm{E}-05$ \\
\hline N & 3.479099 & -0.54718 & $-1.7 \mathrm{E}-05$ \\
\hline C & -1.81914 & 0.87625 & 0.000022 \\
\hline H & 3.776196 & -1.51122 & 0.000004 \\
\hline H & -3.60544 & -1.81049 & $-4.9 \mathrm{E}-05$ \\
\hline O & -2.84184 & 1.346705 & 0.000021 \\
\hline H & -3.09638 & -1.52142 & $-4.8 \mathrm{E}-05$ \\
\hline H & -3.60517 & -1.81066 & -0.76404 \\
\hline O & & & \\
\hline
\end{tabular}

\begin{tabular}{|l|l|}
\hline S3.3 & M06-2X/6-311++G (d, p) \\
\hline Enthalpy & -399.884366 a. u \\
\hline & Cartesian Coordinates (Angstroms) \\
\hline
\end{tabular}




\begin{tabular}{|l|l|l|l|}
\hline & $x$ & $y$ & $z$ \\
\hline C & -0.33351 & 1.240422 & $-4.1 \mathrm{E}-05$ \\
\hline C & 1.025833 & 1.232931 & -0.00003 \\
\hline C & 1.743827 & $-3 \mathrm{E}-06$ & 0.000013 \\
\hline C & 1.025822 & -1.23293 & 0.000045 \\
\hline C & -0.33352 & -1.24041 & 0.000035 \\
\hline C & -1.03858 & 0.000011 & $-8 \mathrm{E}-06$ \\
\hline H & -0.87812 & 2.176577 & $-7.4 \mathrm{E}-05$ \\
\hline H & 1.573274 & 2.167557 & $-5.5 \mathrm{E}-05$ \\
\hline H & 1.57325 & -2.16756 & 0.000078 \\
\hline H & -0.87815 & -2.17656 & 0.00006 \\
\hline N & 3.07389 & -0.00001 & 0.000023 \\
\hline C & -2.40261 & 0.000001 & $-1.8 \mathrm{E}-05$ \\
\hline H & 3.600661 & 0.861047 & 0.000001 \\
\hline H & 3.600658 & -0.86107 & 0.000052 \\
\hline O & -3.52904 & $-5 \mathrm{E}-06$ & $-2.6 \mathrm{E}-05$ \\
\hline
\end{tabular}

\begin{tabular}{|c|c|c|c|}
\hline $\mathrm{H}_{2} \mathrm{O}$ & \multicolumn{3}{|c|}{ M06-2X/6-311++G (d, p) } \\
\hline \multirow[t]{3}{*}{ Enthalpy } & \multicolumn{3}{|c|}{-76.395508} \\
\hline & \multicolumn{3}{|c|}{ Cartesian Coordinates (Angstroms) } \\
\hline & $x$ & $y$ & $z$ \\
\hline $\mathrm{O}$ & 0 & 0.116606 & 0 \\
\hline $\mathrm{H}$ & 0.761538 & -0.46643 & 0 \\
\hline
\end{tabular}




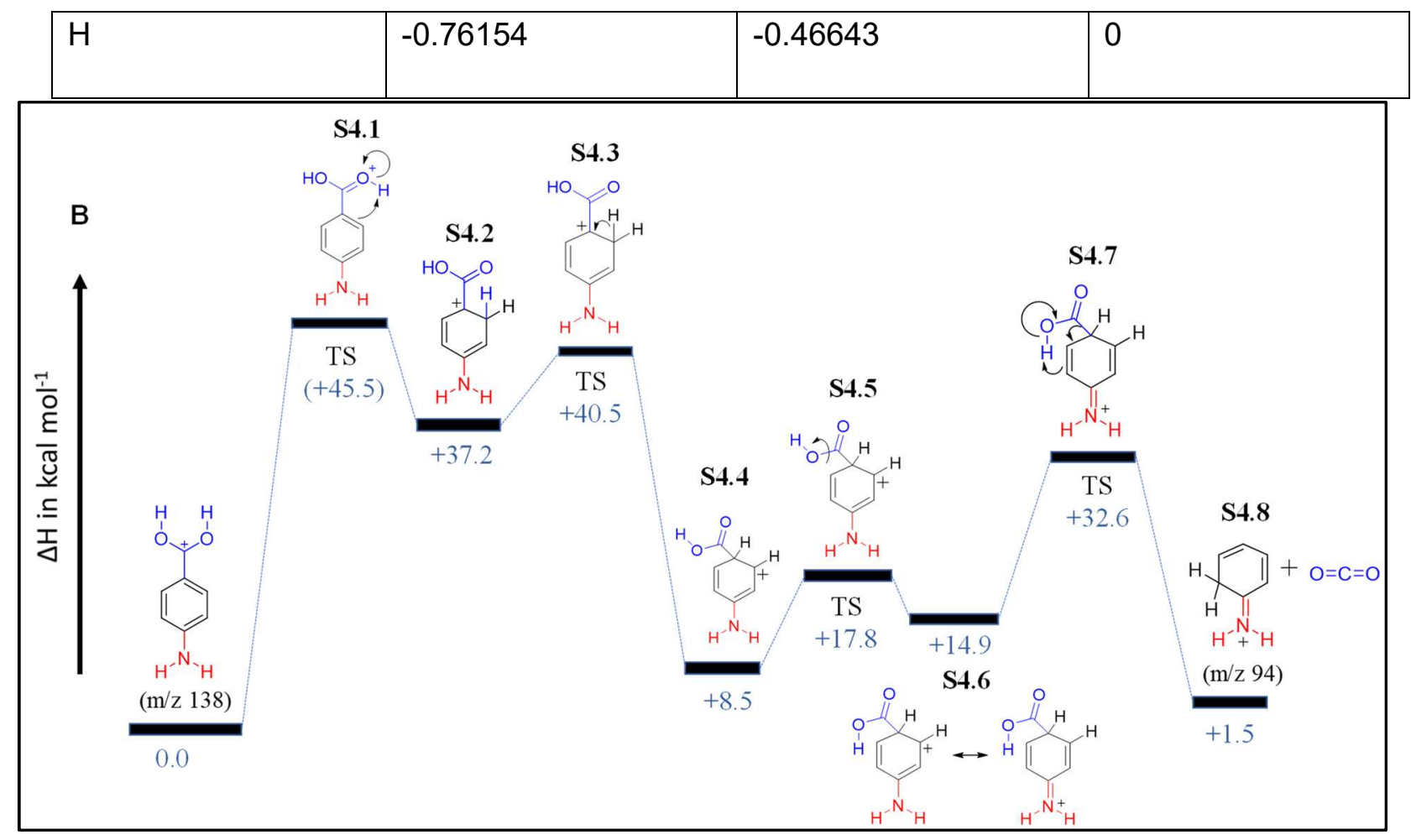

Figure S4. Calculated potential energy surface for the loss of $\mathrm{CO}_{2}$ from the O-protomer of protonated 4aminobenzoic acid.

\begin{tabular}{|l|l|l|l|}
\hline S4.1 & \multicolumn{2}{|l|}{ M06-2X/6-311++G $(d, p)$} & \multicolumn{2}{l|}{} \\
\hline Enthalpy & \multicolumn{2}{|l|}{-476.233981 a.u } & \\
\hline & \multicolumn{2}{|l|}{ Cartesian Coordinates (Angstroms) } \\
\hline & $x$ & $y$ & 0.382702 \\
\hline C & -0.02652 & -1.09794 & 0.254544 \\
\hline C & 1.375598 & -1.14767 & -0.02809 \\
\hline C & 2.111674 & 0.017407 & -0.09875 \\
\hline C & 1.401328 & 1.238974 & 0.013677 \\
\hline C & 0.014229 & 1.311087 & 0.248587 \\
\hline C & -0.70371 & 0.160168 & 0.448658 \\
\hline H & 1.89544 & -2.07965 & -0.30383 \\
\hline H & 1.955863 & 2.148967 & \\
\hline
\end{tabular}




\begin{tabular}{|l|l|l|l|}
\hline $\mathrm{H}$ & -0.47899 & 2.259494 & -0.17049 \\
\hline $\mathrm{N}$ & 3.451314 & -0.01753 & -0.18601 \\
\hline $\mathrm{C}$ & -2.14138 & -0.07339 & -0.07874 \\
\hline $\mathrm{O}$ & -3.08555 & 0.793579 & 0.17849 \\
\hline $\mathrm{O}$ & -2.36014 & -1.13705 & -0.63052 \\
\hline $\mathrm{H}$ & 3.961197 & -0.88538 & -0.15462 \\
\hline $\mathrm{H}$ & 3.981258 & 0.819354 & -0.36732 \\
\hline $\mathrm{H}$ & -2.78158 & 1.53289 & 0.720501 \\
\hline $\mathrm{H}$ & -0.46009 & -1.85095 & 1.055635 \\
\hline $\mathrm{H}$ & -0.85411 & -1.52611 & -0.47377 \\
\hline
\end{tabular}

\begin{tabular}{|l|l|l|l|}
\hline S4.2 & \multicolumn{2}{|l|}{ M06-2X/6-311++G (d, p) } \\
\hline Enthalpy & \multicolumn{2}{|l|}{-476.247173 a.u } & \\
\hline & \multicolumn{2}{|l|}{ Cartesian Coordinates (Angstroms) } \\
\hline & $\mathrm{x}$ & $\mathrm{y}$ & \\
\hline C & -2.2242 & 0.081478 & -0.04338 \\
\hline O & -2.95057 & -0.96965 & 0.329049 \\
\hline H & -2.44546 & -1.63576 & 0.808361 \\
\hline O & -2.70718 & 1.086513 & -0.46365 \\
\hline C & -0.02443 & -1.20399 & -0.14095 \\
\hline C & -0.71938 & -0.03845 & 0.043392 \\
\hline C & 0.015779 & 1.210631 & 0.19233 \\
\hline H & -0.3382 & 1.874001 & -0.63039 \\
\hline
\end{tabular}




\begin{tabular}{|l|l|l|l|}
\hline H & -0.36016 & 1.798623 & 1.049908 \\
\hline C & 1.378164 & -1.18991 & -0.1656 \\
\hline C & 1.466575 & 1.165159 & 0.189006 \\
\hline C & 2.165041 & -0.02219 & -0.00156 \\
\hline N & 3.516128 & -0.08504 & -0.0251 \\
\hline H & 4.001396 & -0.95393 & -0.1771 \\
\hline H & 4.080333 & 0.743785 & 0.068151 \\
\hline H & -0.53127 & -2.1476 & -0.31104 \\
\hline H & 1.893762 & -2.1326 & -0.33013 \\
\hline H & 2.003458 & 2.097478 & 0.335321 \\
\hline
\end{tabular}

\begin{tabular}{|l|l|l|l|}
\hline S4.3 & \multicolumn{2}{|l|}{ M06-2X/6-311++G $(\mathrm{d}, \mathrm{p})$} \\
\hline Enthalpy & \multicolumn{2}{|l|}{$-476.242042 \mathrm{a} . \mathrm{u}$} & \multicolumn{2}{l|}{} \\
\hline & \multicolumn{4}{|l|}{ Cartesian Coordinates (Angstroms) } \\
\hline & $\mathrm{X}$ & $\mathrm{y}$ & \\
\hline C & 2.147805 & 0.005828 & 0.015425 \\
\hline C & 1.444922 & -1.18545 & 0.220943 \\
\hline H & 1.974487 & -2.11302 & 0.403437 \\
\hline $\mathrm{N}$ & 3.490759 & 0.053513 & 0.013083 \\
\hline H & 3.987479 & 0.920399 & -0.11748 \\
\hline H & 4.04597 & -0.77547 & 0.156043 \\
\hline C & 1.386865 & 1.196956 & -0.18741 \\
\hline C & 0.030203 & -1.20449 & 0.18438 \\
\hline
\end{tabular}




\begin{tabular}{|l|l|l|l|}
\hline C & -0.71147 & 0.026774 & 0.020968 \\
\hline C & 0.012207 & 1.209551 & -0.19392 \\
\hline C & -2.22438 & -0.08762 & -0.02784 \\
\hline O & -2.73264 & -1.079 & -0.44506 \\
\hline O & -2.91401 & 0.96404 & 0.40041 \\
\hline H & -2.38044 & 1.617248 & 0.86751 \\
\hline H & 1.920446 & 2.125057 & -0.36694 \\
\hline H & -0.5105 & 2.139233 & -0.39038 \\
\hline H & -0.51683 & -2.095 & 0.479836 \\
\hline H & -0.29962 & -1.04261 & -0.96173 \\
\hline
\end{tabular}

\begin{tabular}{|l|l|l|l|}
\hline S4.4 & \multicolumn{2}{|l|}{ M06-2X/6-311++G $(\mathrm{d}, \mathrm{p})$} \\
\hline Enthalpy & \multicolumn{2}{|l|}{-476.293029 a.u } & \multicolumn{2}{l|}{} \\
\hline & \multicolumn{2}{|l|}{ Cartesian Coordinates (Angstroms) } \\
\hline & $\mathrm{x}$ & $\mathrm{y}$ & \\
\hline & 1.315687 & -1.26562 & 0.016546 \\
\hline C & 2.048576 & -0.02761 & -0.09356 \\
\hline C & 1.383394 & 1.235764 & 0.116445 \\
\hline C & 1.835067 & -2.19895 & -0.1663 \\
\hline H & 1.949949 & 2.151666 & -0.0038 \\
\hline H & 3.322728 & -0.04958 & -0.39993 \\
\hline N & -2.07501 & 0.130958 & -0.11896 \\
\hline C & & & \\
\hline
\end{tabular}




\begin{tabular}{|l|l|l|l|}
\hline H & 3.812951 & -0.91994 & -0.56374 \\
\hline H & 3.860016 & 0.803943 & -0.48747 \\
\hline O & -2.65832 & -1.06104 & -0.20765 \\
\hline H & -3.52464 & -0.95483 & -0.63011 \\
\hline O & -2.52349 & 1.162862 & -0.50265 \\
\hline C & -0.71736 & 0.019454 & 0.609087 \\
\hline C & 0.078367 & 1.25839 & 0.422069 \\
\hline C & 0.012015 & -1.24473 & 0.332843 \\
\hline H & -0.44746 & 2.199267 & 0.539163 \\
\hline H & -1.01499 & -0.02012 & 1.674463 \\
\hline H & -0.5496 & -2.16821 & 0.412832 \\
\hline
\end{tabular}

\begin{tabular}{|l|l|l|l|}
\hline S4.5 & \multicolumn{2}{|l|}{ M06-2X/6-311++G $(\mathrm{d}, \mathrm{p})$} \\
\hline Enthalpy & \multicolumn{2}{l|}{-476.279619 a.u } & \\
\hline & \multicolumn{2}{|l|}{ Cartesian Coordinates (Angstroms) } \\
\hline & $\mathrm{x}$ & $\mathrm{y}$ & \\
\hline $\mathrm{C}$ & 1.236936 & -1.28827 & -0.05931 \\
\hline C & 2.0069 & -0.06809 & -0.08362 \\
\hline C & 1.398732 & 1.187667 & 0.286383 \\
\hline H & 1.717854 & -2.21748 & -0.34223 \\
\hline H & 1.999547 & 2.089194 & 0.255217 \\
\hline N & 3.264687 & -0.09849 & -0.45192 \\
\hline C & -2.01664 & 0.19011 & -0.19741 \\
\hline
\end{tabular}




\begin{tabular}{|l|l|l|l|}
\hline H & 3.716001 & -0.96279 & -0.72535 \\
\hline H & 3.826516 & 0.743406 & -0.48533 \\
\hline O & -2.85803 & -0.86869 & -0.14552 \\
\hline H & -3.40722 & -0.88258 & 0.648224 \\
\hline O & -2.15978 & 1.124738 & -0.90753 \\
\hline C & -0.74621 & 0.007085 & 0.681297 \\
\hline C & 0.104547 & 1.223277 & 0.632718 \\
\hline C & -0.05584 & -1.2543 & 0.297515 \\
\hline H & -0.36719 & 2.168716 & 0.877099 \\
\hline H & -1.11852 & -0.11924 & 1.712074 \\
\hline H & -0.64779 & -2.16304 & 0.302656 \\
\hline
\end{tabular}

\begin{tabular}{|l|l|l|l|}
\hline S4.6 & \multicolumn{2}{|l|}{ M06-2X/6-311++G $(\mathrm{d}, \mathrm{p})$} \\
\hline Enthalpy & \multicolumn{2}{l|}{-476.282718 a.u } & \\
\hline & \multicolumn{2}{|l|}{ Cartesian Coordinates (Angstroms) } \\
\hline & $\mathrm{x}$ & $\mathrm{y}$ & \\
\hline $\mathrm{C}$ & -1.21221 & 1.297791 & -0.0797 \\
\hline C & -1.9713 & 0.068516 & -0.0745 \\
\hline C & -1.36982 & -1.15888 & 0.387433 \\
\hline H & -1.69573 & 2.211108 & -0.40642 \\
\hline H & -1.96817 & -2.06238 & 0.402524 \\
\hline N & -3.21326 & 0.070363 & -0.49077 \\
\hline C & 1.964588 & -0.19615 & -0.25584 \\
\hline
\end{tabular}




\begin{tabular}{|l|l|l|l|}
\hline H & -3.65881 & 0.914958 & -0.8284 \\
\hline H & -3.76744 & -0.77749 & -0.50414 \\
\hline O & 3.026341 & 0.591396 & -0.08902 \\
\hline H & 2.995559 & 1.124169 & 0.713048 \\
\hline O & 1.88879 & -1.00253 & -1.12104 \\
\hline C & 0.773994 & 0.04615 & 0.731561 \\
\hline C & -0.08273 & -1.16829 & 0.758074 \\
\hline C & 0.071355 & 1.288955 & 0.306433 \\
\hline H & 0.385627 & -2.09538 & 1.070343 \\
\hline H & 1.19833 & 0.198759 & 1.735163 \\
\hline H & 0.639129 & 2.214172 & 0.292889 \\
\hline
\end{tabular}

\begin{tabular}{|l|l|l|l|}
\hline S4.7 & \multicolumn{2}{|l|}{ M06-2X/6-311++G (d, p) } \\
\hline Enthalpy & \multicolumn{2}{|l|}{-476.254634 a.u } & \\
\hline & \multicolumn{2}{|l|}{ Cartesian Coordinates (Angstroms) } \\
\hline & $\mathrm{x}$ & $\mathrm{y}$ & \\
\hline & -1.01147 & -0.99631 & 0.850163 \\
\hline C & -1.6839 & 0.016095 & 0.117883 \\
\hline C & -1.08594 & 0.489409 & -1.08729 \\
\hline C & -1.44661 & -1.36537 & 1.77136 \\
\hline H & -1.60307 & 1.242521 & -1.67192 \\
\hline H & & & \\
\hline
\end{tabular}




\begin{tabular}{|l|l|l|l|}
\hline N & -2.83011 & 0.547786 & 0.566737 \\
\hline C & 1.919453 & 0.607161 & 0.38552 \\
\hline H & -3.26015 & 0.214776 & 1.415753 \\
\hline H & -3.35595 & 1.20641 & 0.013391 \\
\hline O & 1.041911 & 1.458264 & 0.485778 \\
\hline H & 0.181794 & 1.236269 & -0.08191 \\
\hline O & 2.955224 & 0.165096 & 0.546158 \\
\hline C & 0.746268 & -1.07204 & -0.83987 \\
\hline C & 0.049387 & -0.13467 & -1.60431 \\
\hline C & 0.16588 & -1.52115 & 0.376929 \\
\hline H & 0.432778 & 0.179385 & -2.5689 \\
\hline H & 1.618984 & -1.57097 & -1.24126 \\
\hline H & 0.667863 & -2.29543 & 0.946697 \\
\hline
\end{tabular}

\begin{tabular}{|l|l|l|l|}
\hline S4.8 & \multicolumn{2}{|l|}{ M06-2X/6-311++G (d, p) } \\
\hline Enthalpy & \multicolumn{2}{|l|}{-476.749581 a.u } & \\
\hline & \multicolumn{2}{|l|}{ Cartesian Coordinates (Angstroms) } \\
\hline & $\mathrm{x}$ & $\mathrm{y}$ & \\
\hline & 0.208211 & -1.25697 & 0.00001 \\
\hline C & 0.962524 & 0.031457 & 0.000002 \\
\hline C & 0.264148 & 1.26522 & 0.000012 \\
\hline C & 0.522552 & -1.84883 & 0.871273 \\
\hline H & 0.819229 & 2.195584 & 0.000014 \\
\hline H & & & \\
\hline
\end{tabular}




\begin{tabular}{|l|l|l|l|}
\hline N & 2.276155 & -0.00443 & $-8 \mathrm{E}-06$ \\
\hline $\mathrm{H}$ & 2.788276 & -0.87738 & $-2.1 \mathrm{E}-05$ \\
\hline $\mathrm{H}$ & 2.823878 & 0.847175 & -0.00003 \\
\hline $\mathrm{C}$ & -1.88983 & 0.053564 & $-8 \mathrm{E}-06$ \\
\hline $\mathrm{C}$ & -1.09872 & 1.259405 & $-2 \mathrm{E}-06$ \\
\hline $\mathrm{C}$ & -1.27685 & -1.13872 & $-6 \mathrm{E}-06$ \\
\hline $\mathrm{H}$ & -1.6179 & 2.212237 & 0.000002 \\
\hline $\mathrm{H}$ & -2.9687 & 0.132066 & $-2.1 \mathrm{E}-05$ \\
\hline $\mathrm{H}$ & -1.83993 & -2.0647 & $-8 \mathrm{E}-06$ \\
\hline $\mathrm{H}$ & 0.522596 & -1.84888 & -0.8712 \\
\hline
\end{tabular}

\begin{tabular}{|l|l|l|l|}
\hline $\mathrm{CO}_{2}$ & \multicolumn{2}{|l|}{$\mathrm{M} 06-2 \mathrm{X} / 6-311++\mathrm{G}(\mathrm{d}, \mathrm{p})$} \\
\hline Enthalpy & \multicolumn{2}{l|}{-188.559337} & $\mathrm{y}$ \\
\hline & \multicolumn{2}{|l|}{ Cartesian Coordinates (Angstroms) } \\
\hline & $\mathrm{x}$ & 0 & $-4 \mathrm{E}-06$ \\
\hline $\mathrm{C}$ & 0 & 0 & 1.154613 \\
\hline $\mathrm{O}$ & 0 & 0 & -1.15461 \\
\hline $\mathrm{O}$ & 0 & 0 & \\
\hline
\end{tabular}




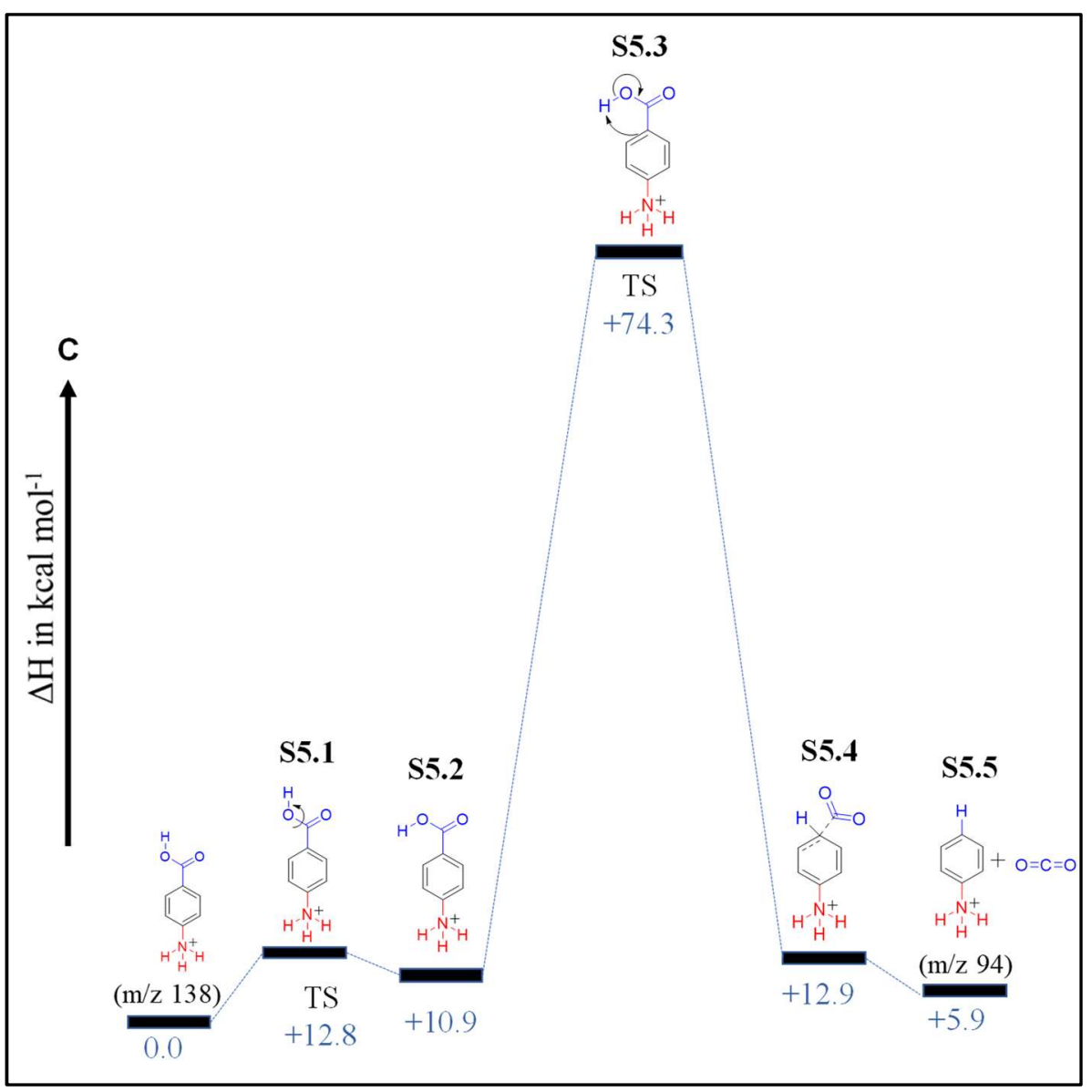

Figure S5. Calculated potential energy surface for the loss of $\mathrm{CO}_{2}$ from the $\mathrm{N}$ - protomer of protonated 4-aminobenzoic acid.

\begin{tabular}{|c|c|c|c|}
\hline $\begin{array}{l}\text { N-protomer of pro- } \\
\text { tonated } \\
\text { 4-aminobenzoic } \\
\text { acid }\end{array}$ & \multicolumn{3}{|c|}{$M 06-2 X / 6-311++G(d, p)$} \\
\hline \multirow[t]{3}{*}{ Enthalpy } & \multicolumn{3}{|c|}{-476.308057 a.u } \\
\hline & \multicolumn{3}{|c|}{ Cartesian Coordinates (Angstroms) } \\
\hline & $\mathrm{x}$ & $\mathrm{y}$ & z \\
\hline $\mathrm{C}$ & -1.3315 & 1.223104 & -0.00323 \\
\hline
\end{tabular}




\begin{tabular}{|l|l|l|l|l|}
\hline C & & 0.059665 & 1.195014 & -0.00115 \\
\hline C & & 0.725301 & -0.02693 & 0.000175 \\
\hline C & & 0.018329 & -1.22833 & -0.00109 \\
\hline C & & -1.36871 & -1.21703 & -0.00349 \\
\hline C & & -2.00364 & 0.014877 & -0.00451 \\
\hline H & -1.86089 & 2.17 & -0.00434 \\
\hline H & & 0.624266 & 2.11834 & -0.00189 \\
\hline H & & 0.568921 & -2.16146 & -0.00169 \\
\hline H & & -1.92903 & -2.14592 & -0.0048 \\
\hline C & & 2.225812 & -0.11121 & 0.001084 \\
\hline O & & 2.82114 & -1.14798 & 0.001735 \\
\hline O & & 2.795768 & 1.096407 & 0.001232 \\
\hline N & & -3.49327 & 0.032828 & 0.005679 \\
\hline H & & -3.84925 & 0.979558 & -0.15989 \\
\hline H & & -3.87851 & -0.57367 & -0.72648 \\
\hline H & & -0.28586 & 0.906822 \\
\hline H & & 0.984792 & 0.001969 \\
\hline
\end{tabular}

\begin{tabular}{|l|l|}
\hline S5.1 & M06-2X/6-311++G $(\mathrm{d}, \mathrm{p})$ \\
\hline Enthalpy & -476.287656 a.u \\
\hline & Cartesian Coordinates (Angstroms) \\
\hline
\end{tabular}




\begin{tabular}{|c|c|c|c|}
\hline & $\mathrm{x}$ & $y$ & $z$ \\
\hline$C$ & -1.37145 & 1.212153 & -0.09721 \\
\hline$C$ & 0.014583 & 1.23205 & -0.0941 \\
\hline C & 0.732315 & 0.037083 & -0.015 \\
\hline C & 0.07072 & -1.1836 & 0.072395 \\
\hline C & -1.3212 & -1.22079 & 0.079841 \\
\hline C & -2.00043 & -0.02062 & -0.00727 \\
\hline $\mathrm{H}$ & -1.9373 & 2.135577 & -0.16112 \\
\hline $\mathrm{H}$ & 0.556811 & 2.168553 & -0.14658 \\
\hline $\mathrm{H}$ & 0.637769 & -2.10189 & 0.157337 \\
\hline $\mathrm{H}$ & -1.84432 & -2.16789 & 0.158977 \\
\hline C & 2.238632 & 0.12504 & 0.022192 \\
\hline 0 & 2.887741 & -1.0664 & -0.07082 \\
\hline 0 & 2.797618 & 1.157982 & 0.201837 \\
\hline $\mathrm{N}$ & -3.48966 & -0.04426 & 0.014184 \\
\hline $\mathrm{N}$ & -3.84185 & -1.00595 & -0.02558 \\
\hline $\mathrm{N}$ & -3.85922 & 0.385243 & 0.870114 \\
\hline $\mathrm{N}$ & 2.937529 & -1.38634 & -0.97912 \\
\hline $\mathrm{N}$ & -3.88373 & 0.461911 & -0.78651 \\
\hline
\end{tabular}

\begin{tabular}{|l|l|}
\hline S5.2 & M06-2X/6-311++G (d, p) \\
\hline Enthalpy & -476.290712 a.u \\
\hline & Cartesian Coordinates (Angstroms) \\
\hline
\end{tabular}




\begin{tabular}{|c|c|c|c|}
\hline & $x$ & $y$ & $z$ \\
\hline$C$ & -1.35193 & -1.20211 & 0.171725 \\
\hline$C$ & 0.033703 & -1.20521 & 0.162758 \\
\hline$C$ & 0.738814 & -0.00951 & 0.012477 \\
\hline C & 0.058338 & 1.192193 & -0.15661 \\
\hline C & -1.33432 & 1.211998 & -0.1662 \\
\hline C & -1.99891 & 0.013206 & 0.004481 \\
\hline $\mathrm{H}$ & -1.90439 & -2.12755 & 0.295911 \\
\hline $\mathrm{H}$ & 0.58818 & -2.13079 & 0.260904 \\
\hline $\mathrm{H}$ & 0.59292 & 2.121057 & -0.32041 \\
\hline $\mathrm{H}$ & -1.86762 & 2.145128 & -0.31292 \\
\hline C & 2.248336 & -0.11277 & -0.03628 \\
\hline 0 & 2.947387 & 0.973581 & 0.316117 \\
\hline 0 & 2.779324 & -1.11561 & -0.3956 \\
\hline $\mathrm{N}$ & -3.488 & 0.016506 & -0.00102 \\
\hline $\mathrm{H}$ & -3.85325 & 0.957026 & -0.18169 \\
\hline $\mathrm{H}$ & -3.86032 & -0.60333 & -0.72945 \\
\hline $\mathrm{H}$ & 2.410735 & 1.626849 & 0.776273 \\
\hline $\mathrm{H}$ & -3.86809 & -0.29453 & 0.900252 \\
\hline
\end{tabular}

S5.3 M06-2X/6-311++G (d, p) 


\begin{tabular}{|c|c|c|c|}
\hline Enthalpy & \multicolumn{3}{|c|}{-476.189603 a.u } \\
\hline & \multicolumn{3}{|c|}{ Cartesian Coordinates (Angstroms) } \\
\hline & $\mathrm{x}$ & $\mathrm{y}$ & $z$ \\
\hline$C$ & 1.399199 & 1.219596 & 0.082086 \\
\hline C & 0.021055 & 1.204485 & 0.271168 \\
\hline C & -0.68406 & -0.00125 & 0.367992 \\
\hline C & 0.020321 & -1.20618 & 0.264935 \\
\hline C & 1.399046 & -1.22041 & 0.075607 \\
\hline C & 2.046597 & -0.00054 & -0.01186 \\
\hline $\mathrm{H}$ & 1.941139 & 2.157259 & 0.01085 \\
\hline $\mathrm{H}$ & -0.50023 & 2.152893 & 0.347897 \\
\hline $\mathrm{H}$ & -0.50093 & -2.15498 & 0.336805 \\
\hline $\mathrm{H}$ & 1.94025 & -2.15814 & -0.00047 \\
\hline C & -2.61913 & 0.001138 & -0.24993 \\
\hline $\mathrm{O}$ & -3.11312 & -0.00547 & 0.892365 \\
\hline $\mathrm{O}$ & -2.72711 & 0.007614 & -1.39519 \\
\hline $\mathrm{N}$ & 3.518587 & 0.001131 & -0.24487 \\
\hline $\mathrm{H}$ & 3.968586 & 0.785937 & 0.237528 \\
\hline $\mathrm{H}$ & 3.947205 & -0.862 & 0.104459 \\
\hline $\mathrm{H}$ & 3.741761 & 0.078165 & -1.24385 \\
\hline $\mathrm{H}$ & -1.9442 & -0.00524 & 1.143571 \\
\hline
\end{tabular}

S5.4

M06-2X/6-311++G (d, p) 


\begin{tabular}{|c|c|c|c|}
\hline Enthalpy & \multicolumn{3}{|c|}{-476.328709 a.u } \\
\hline & \multicolumn{3}{|c|}{ Cartesian Coordinates (Angstroms) } \\
\hline & $x$ & $y$ & $z$ \\
\hline C & -2.10705 & 1.067424 & 0.453505 \\
\hline C & -3.09755 & 0.115719 & 0.673473 \\
\hline C & -2.92381 & -1.18888 & 0.223796 \\
\hline C & -1.76088 & -1.55677 & -0.44679 \\
\hline C & -0.75918 & -0.61993 & -0.67397 \\
\hline C & -0.96262 & 0.670888 & -0.21557 \\
\hline $\mathrm{H}$ & -2.23591 & 2.0876 & 0.799532 \\
\hline $\mathrm{H}$ & -4.00188 & 0.398344 & 1.196838 \\
\hline $\mathrm{H}$ & -1.62891 & -2.57379 & -0.79317 \\
\hline $\mathrm{H}$ & 0.153461 & -0.89398 & -1.19161 \\
\hline C & 3.613382 & -0.25608 & 0.206053 \\
\hline $\mathrm{O}$ & 4.602161 & -0.75102 & 0.495192 \\
\hline $\mathrm{O}$ & 2.60326 & 0.246631 & -0.09034 \\
\hline $\mathrm{N}$ & 0.096109 & 1.679922 & -0.47852 \\
\hline $\mathrm{H}$ & 1.032747 & 1.268496 & -0.35106 \\
\hline $\mathrm{H}$ & 0.036683 & 2.046181 & -1.43421 \\
\hline $\mathrm{H}$ & 0.012553 & 2.473199 & 0.163052 \\
\hline $\mathrm{H}$ & -3.69867 & -1.92465 & 0.398453 \\
\hline
\end{tabular}

\begin{tabular}{|l|l}
\hline S5.5 & M06-2X/6-311++G (d, p)
\end{tabular} 


\begin{tabular}{|l|l|l|l|}
\hline Enthalpy & \multicolumn{2}{|l|}{-287.758122 a.u } & \multicolumn{2}{l|}{} \\
\hline & \multicolumn{2}{|l|}{ Cartesian Coordinates (Angstroms) } \\
\hline & X & \multicolumn{2}{l|}{} \\
\hline C & -1.2225 & -1.20819 & 0.001521 \\
\hline C & 0.168388 & -1.21916 & -0.00592 \\
\hline C & 0.823694 & -0.00051 & -0.00981 \\
\hline C & 0.169131 & 1.21902 & -0.00598 \\
\hline C & -1.22133 & 1.208781 & 0.001575 \\
\hline C & -1.91141 & 0.000354 & 0.00594 \\
\hline H & -1.76256 & -2.14621 & 0.001043 \\
\hline H & 0.712959 & -2.15751 & -0.00961 \\
\hline H & 0.714732 & 2.156749 & -0.00971 \\
\hline H & -1.76096 & 2.147074 & 0.001181 \\
\hline H & -2.99413 & 0.000837 & 0.009454 \\
\hline N & 2.313888 & $-7.6 \mathrm{E}-05$ & 0.008088 \\
\hline H & 2.68128 & 0.057879 & -0.564585 \\
\hline H & 2.68653 & -0.8546 & \\
\hline H & 2.689034 & 0.794512 & \\
\hline
\end{tabular}

